\title{
Stability of an adiabatic circuit with inductive load using 1D-capacitor array between the power supply and ground
}

\author{
Shunji Nakata ${ }^{a)}$ \\ NTT Microsystem Integration Laboratories, \\ 3-1 Morinosato Wakamiya, Atsugi-shi, Kanagawa 243-0198, Japan \\ a)nakata@aecl.ntt.co.jp
}

\begin{abstract}
The stability of a new adiabatic circuit with inductive load is discussed. The adiabatic circuit generates quasi-sinusoidal waveform current with multiple power-supply voltages. SPICE simulation shows that this circuit is stable after damping oscillation. For the analytical discussion, we derive a matrix that connects charge, current, and voltage in the circuit. By using matrix theory and a physical consideration, it is proved that the absolute value of the eigen value of the matrix, which connects the initial voltage and current deviations from the equally-divided-stepwise mode with those after the charge-recycling process, is smaller than 1 . Therefore, the voltage and current deviations become zero after many charge-recycling processes. The circuit might be useful for a DC-AC inverter in the power electronics.
\end{abstract}

Keywords: adiabatic circuit, charge recycling, asymmetric 1D capacitor array, inductive load, stability, DC-AC inverter, power electronics Classification: Integrated circuits

\section{References}

[1] Power Semiconductor Device and Power IC Handbook, Edited by The Institute of Electrical Engineers of Japan, Corona Publishing Co., LTD, Tokyo, pp. 400-402, 1996.

[2] S. Nakata, "Adiabatic Charging Reversible Logic using a Switched Capacitor Regenerator," IEICE Trans. Electron., vol. E87-C, no. 11, pp. 18371846, 2004.

[3] S. Nakata, "Stability of adiabatic circuit using asymmetric 1D-capacitor array between the power supply and ground," IEICE Electron. Express, vol. 4, no. 5, pp. 165-171, 2007.

[4] T. Imamura, Physics and Matrix, Iwanami Shoten, Tokyo, 1994. 


\section{Introduction}

When using a DC-AC inverter, pulse width modulation (PWM) is usually used to generate a sinusoidal waveform. However, the generated waveform is not perfect; there is a ripple in it. To smoothen the waveform, a 3-level inverter was developed, which has three levels of voltage: $\mathrm{V}_{\mathrm{DD}}, \mathrm{V}_{\mathrm{DD}} / 2$, and GND. The combination of a 3-level inverter and PWM produces a better waveform. Further smoothing of the waveform would require a multi-level inverter [1]. However, it has been thought to be difficult to realize multiple power-supply voltages. In this article, we propose a multi-level inverter, whose multi-level power supply is spontaneously generated by an adiabatic circuit with the 1D capacitor array. We discuss the stability of the circuit using SPICE simulation and prove it by an analytical method.

\section{Circuit structure and stability}

The switched capacitor inverter with the $1 \mathrm{D}$ capacitor array is shown in Fig. 1 (a). For simplicity, we show a four-step waveform circuit. The circuit structure is almost the same as in our previous work $[2,3]$. The different point is that the load is changed from capacitive to inductive. As in the previous work, tank capacitors $C_{i}$ are connected in series between the power supply $V$ and ground. $V_{\text {out }}$ is output voltage, and $V_{C i}$ is the voltage of the node between tank capacitor $C_{i}$ and $C_{i+1}$. $L$ is load inductance, and
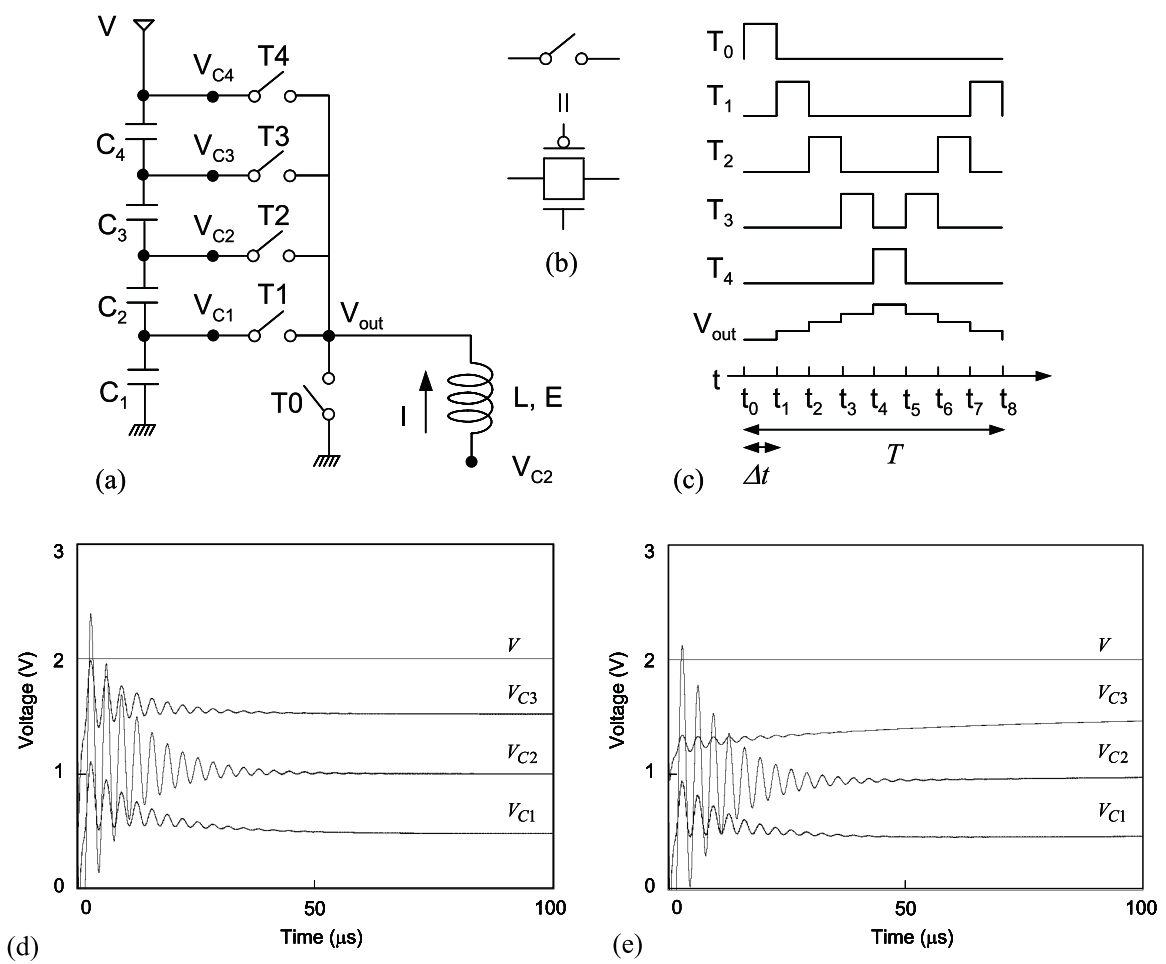

Fig. 1. Multi-level inverter with the 1D capacitor array. (a) Circuit. (b) Switching transistor circuit. (c) Timing chart. (d) Simulation results of voltage change of $V_{C i}$ with $C_{i}=20 \mathrm{pF}$. (e) The same with $C_{1}=C_{2}=C_{3}=20 \mathrm{pF}, C_{4}=200 \mathrm{pF}$. 
$E\left(E=V_{C 2}-V_{\text {out }}\right)$ is inductor voltage. The switching transistor is a parallel connection of a pMOSFET and nMOSFET, as shown in Fig. 1 (b).

First, we discuss the simulation results. We used the $0.25-\mu \mathrm{m}$ design rule. For the pMOS and nMOS transistors, the gate length and the width were 0.25 and $6 \mu \mathrm{m}$, respectively. $V$ was $2 \mathrm{~V}$. Threshold voltages were 0.4 and $-0.4 \mathrm{~V}$ in the nMOS and pMOS transistors, respectively. The pulse time of $\Delta t$ and the period of $T$ in Fig. 1 (c) were $25 \mathrm{~ns}$ and $200 \mathrm{~ns}$, respectively. Load inductance $L$ was $4 \mathrm{mH}$.

Figs. 1 (d) and (e) show the simulation results for (d) $C_{i}=20 \mathrm{pF}$ and (e) $C_{1}=C_{2}=C_{3}=20 \mathrm{pF}, C_{4}=200 \mathrm{pF}$, respectively. It is clear that $V_{C i}$ becomes $i V / 4$ spontaneously after $100 \mu \mathrm{s}$. From the results, we can say that the operation of the switched capacitor inverter is stable and not dependent on $C_{i}$.

Next, we investigate the reason for the stability analytically. Fig. 2 (a) and (b) show $V_{\text {out }}$ and current $I$ which flows in the inductor. From the equation $E=L d I / d t$, we have $\Delta I=E / L \cdot \Delta t$. During period $\mathrm{r}_{2}$ and $\mathrm{r}_{6}$, $E=V_{C 2}-V_{\text {out }}=0$ is satisfied. Therefore, we have $\Delta I=0$ during those

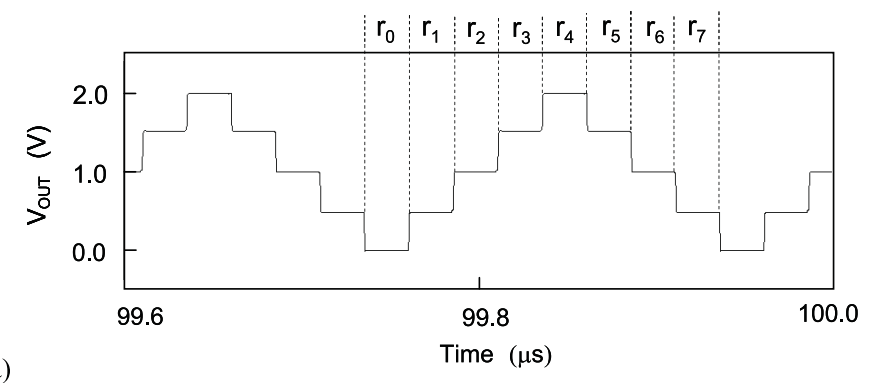

(a)

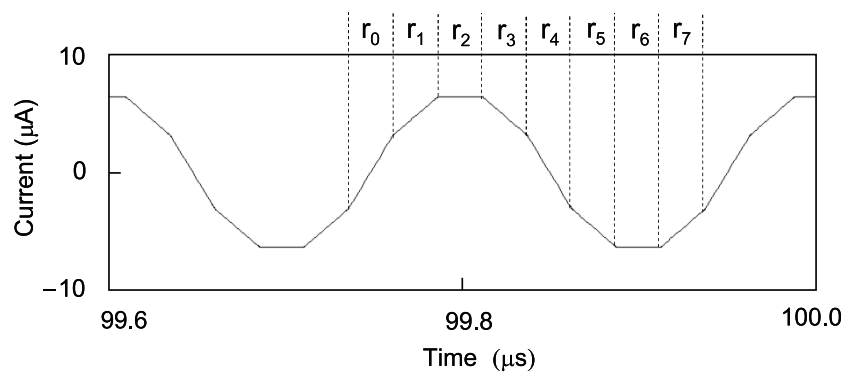

(b)

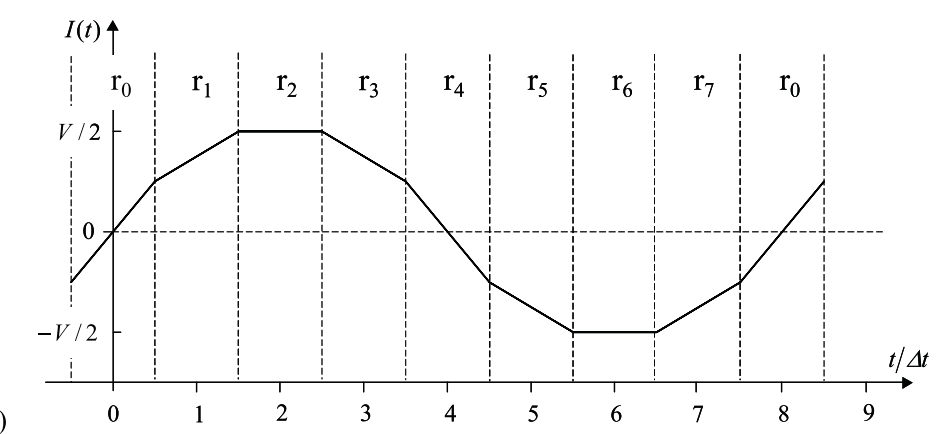

Fig. 2. Simulation results and the ideal $I(t)$ in the equally-divided-stepwise mode. (a) Simulation of $V_{\text {out }}$. (b) Simulation of inductor current. (c) Schematic picture of the ideal $I(t)$. 
periods. During period $\mathrm{r}_{0}, E=1 \mathrm{~V}$. Using $L=4 \mathrm{mH}$ and $\Delta t=25 \mathrm{~ns}$, we calculate $\Delta I$ to be $6.3 \mu \mathrm{A}$. These estimations are consistent with Fig. 2 (b).

Next, for the following discussion, we consider the ideal $i(t)$ curve in the equally-divided-stepwise mode as shown in Fig. 2 (c). This curve is valid from the result in Fig. 2 (b). This curve has the period of $8 \Delta t$ and $I$ is equal to 0 at $t=0$. The $I_{i}(t)$ in the $r_{i}$ region $(0 \leqq i \leqq 7)$ can be written as

$$
I_{i}(t)=a_{i}(t / \Delta t-(i-1 / 2))+b_{i} \text {, when } i-1 / 2 \leq t / \Delta t \leq i+1 / 2 .
$$

To simplify the calculation, we set $L=1$ and $\Delta t=1$. Then, the values of $a_{k}$ and $b_{k}$ can be written using only $V$. Next, we consider the occurrence of deviations. In this case, $a_{k}$ and $b_{k}$ can be written using $V, V_{i}$, and $\varepsilon$, where $V_{i}$ is a deviation from the step voltage $\left(V_{i}=V_{C i}-i V / N\right)$ and $\varepsilon$ is the deviation current at $t=0(\varepsilon=I(0))$.

Here, we define node $N_{C i}$ as the point between $C_{i}$ and $C_{i+1}$, and $Q_{i}$ as the stored charge on $N_{C i}$ as shown in Fig. 3. Then, $\Delta Q_{i}$ and $\Delta \varepsilon$ (the change of $Q_{i}$ and $\varepsilon$ ) after one cycle of $T$ can be derived by considering current flow of $I_{i}(t)$.

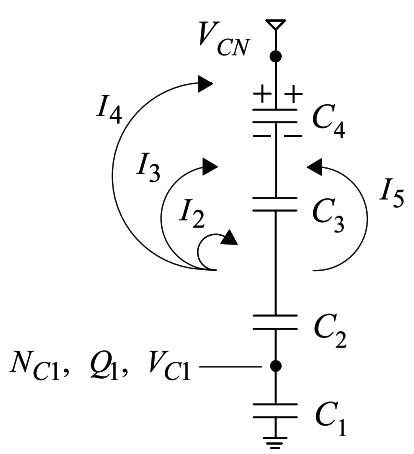

(a) (b)

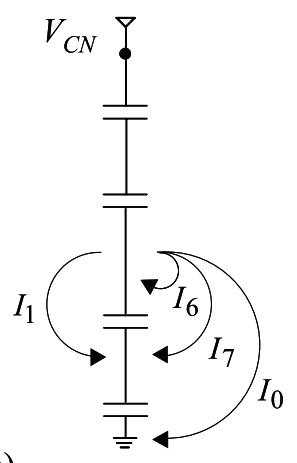

Fig. 3. Current flow directions in $r_{k}$ and definitions of charge $Q_{i}$ in the switched capacitor inverter.

Fig. 3 shows the flow direction of $I_{i}$ in the $r_{i}$ region in Fig. 2 (c). For example, as indicated by arrow $I_{2}$, current flows from $N_{C 2}$ to $N_{C 2}$ itself through the inductor. As indicated by arrow $I_{3}$, it flows from $N_{C 2}$ to $N_{C 3}$ through the inductor.

Then, $\Delta Q_{i}$ on $N_{C i}$ can be calculated by integrating $I(t)$ to $t$. Then, we have

$$
\begin{aligned}
& \Delta Q_{0}=S_{0}, \Delta Q_{1}=S_{1}+S_{7}, \Delta Q_{3}=S_{3}+S_{5}, \text { and } \Delta Q_{4}=S_{4}, \\
& \text { where } S_{i}=\int_{i-1 / 2}^{i+1 / 2} I_{i}(t) d t
\end{aligned}
$$

$S_{i}$ means the charge flow from $N_{C 2}$ to another node in the $r_{i}$ region in Fig. 3. Regarding $\Delta Q_{2}$, using the charge conservation law, we have

$$
\Delta Q_{2}=-\Delta Q_{0}-\Delta Q_{1}-\Delta Q_{3}-\Delta Q_{4}
$$


Then, using (1)-(4), we can calculate $\Delta Q_{i}$ and $\Delta \varepsilon$. From the calculation, we can know that $\Delta Q_{i}$ and $\Delta \varepsilon$ can be expressed as a linear combination of $V_{i}$ and $\varepsilon$. Also, in the case of general $n$, we can calculate $\Delta Q_{i}$ and $\Delta \varepsilon$ similarly.

However, we can derive the characteristic of this linear combination in an easy manner even if we do not calculate $\Delta Q_{i}$ and $\Delta \varepsilon$ directly. From Fig. 2 (c), the equally-divided-stepwise mode $\left(V_{i}=\varepsilon=0\right)$ shows that $I(t)$ is an odd function when we view it from the time at $t=4$. From the odd function characteristic of $I(t)$, we obtain $\Delta Q_{i}=\Delta \varepsilon=0$ easily. If the state is not in the equally-divided-stepwise mode, there is a deviation from $V_{i}=\varepsilon=0$. By considering the 1st order of $V_{i}$ and $\varepsilon$, we can write

$$
\left[\Delta Q_{1}, \cdots, \Delta Q_{N-1}, \Delta \varepsilon\right]^{t}=\boldsymbol{A} \cdot\left[V_{1}, \cdots, V_{N-1}, \varepsilon\right]^{t},
$$

where $\boldsymbol{A}$ is an $N \times N$ matrix. Using $\boldsymbol{Q}=\left[Q_{1}, \ldots, Q_{N-1}\right]^{t}$ and $\boldsymbol{V}=$ $\left[V_{1}, \ldots, V_{N-1}\right]^{t}$, we can rewrite $(5)$ as

$$
\Delta \boldsymbol{q}_{+}=\boldsymbol{A} \boldsymbol{v}_{+}, \text {where } \boldsymbol{q}_{+}=\left[\begin{array}{c}
\boldsymbol{Q} \\
\varepsilon
\end{array}\right], \boldsymbol{v}_{+}=\left[\begin{array}{l}
\boldsymbol{V} \\
\varepsilon
\end{array}\right] .
$$

Equation (6) means that there is a linear relation between $\Delta \boldsymbol{q}_{+}$and $\boldsymbol{v}_{+}$, which has already been pointed out. This is a very important relation for the following discussion.

In the previous paper [3], the following equation was derived.

$$
\Delta \boldsymbol{Q}=\boldsymbol{F} \cdot \Delta \boldsymbol{V}, \text { where } \boldsymbol{F}=\left[\begin{array}{cccc}
C_{1}+C_{2} & -C_{2} & & \\
-C_{2} & C_{2}+C_{3} & -C_{3} & \\
& -C_{3} & \ddots & \\
& & & C_{N-1}+C_{N}
\end{array}\right]
$$

Here, $\boldsymbol{F}$ is an $(N-1) \times(N-1)$ matrix. Using $\boldsymbol{q}_{+}, \boldsymbol{v}_{+}$, and (7), we have

$$
\Delta \boldsymbol{q}_{+}=\boldsymbol{F}_{+} \cdot \Delta \boldsymbol{v}_{+}, \text {where } \boldsymbol{F}_{+}=\left[\begin{array}{cc}
\boldsymbol{F} & \mathbf{0} \\
\mathbf{0} & 1
\end{array}\right] .
$$

Here, $\boldsymbol{F}_{+}$is an $N \times N$ matrix. Using (6) and (8), we have the important equation

$$
\Delta \boldsymbol{q}_{+}=\boldsymbol{F}_{+} \cdot \Delta \boldsymbol{v}_{+}=\boldsymbol{A} \cdot \boldsymbol{v}_{+}
$$

Next, we investigate the characteristic of matrix $\boldsymbol{F}_{+} \cdot \boldsymbol{F}$ is a positivedefinite symmetric matrix so that $\boldsymbol{F}_{+}$is also a positive-definite one. Therefore, there exist a regular matrix $\boldsymbol{P}$ such that $\boldsymbol{P}^{t} \boldsymbol{F}_{+} \boldsymbol{P}=\boldsymbol{I}$ [4]. Next, we consider Jordan matrix theory. In general, for arbitrary matrix $\boldsymbol{K}$, there exists matrix $\boldsymbol{T}$ that transforms $\boldsymbol{T}^{-1} \boldsymbol{K} \boldsymbol{T}$ to a Jordan normal form $\boldsymbol{J} . \boldsymbol{J}$ is written as

$$
\boldsymbol{J}=J_{1} \oplus J_{2} \cdots \oplus J_{r}, \text { where } \boldsymbol{J}_{i}=J\left(\alpha_{i}, n_{i}\right)=\left[\begin{array}{cccc}
\alpha_{i} & 1 & & 0 \\
& \ddots & \ddots & \\
& & \ddots & 1 \\
& & & \alpha_{i}
\end{array}\right] .
$$


Here, $\boldsymbol{J}_{i}$ is an $n_{i} \times n_{i}$ matrix. Therefore, we can transform $\boldsymbol{A}$ and $\boldsymbol{F}_{+}$into $\boldsymbol{J}$ and $\boldsymbol{I}$ as

$$
\boldsymbol{T}^{-1} \boldsymbol{P}^{t} \boldsymbol{A P T}=\boldsymbol{J} \text { and } \boldsymbol{T}^{-1} \boldsymbol{P}^{t} \boldsymbol{F}_{+} \boldsymbol{P} \boldsymbol{T}=\boldsymbol{I} .
$$

Next, for the following discussion, we consider the generalized eigen equation $\boldsymbol{A} \boldsymbol{p}=\lambda \boldsymbol{F}_{+} \boldsymbol{p}$, where $\lambda$ is the generalized eigen value and $\boldsymbol{p}$ is the eigenvector of $\lambda$. Defining $\boldsymbol{q}=(\boldsymbol{P} \boldsymbol{T})^{-1} \boldsymbol{p}$, we have

$$
\boldsymbol{T}^{-1} \boldsymbol{P}^{t}\left(\lambda \boldsymbol{F}_{+}-\boldsymbol{A}\right) \boldsymbol{P} \boldsymbol{T} \boldsymbol{q}=(\lambda \boldsymbol{I}-\boldsymbol{J}) \boldsymbol{q}=0 .
$$

Using $|\lambda \boldsymbol{I}-\boldsymbol{J}|=0$, we have $\lambda=\alpha_{i}$. Regarding $\lambda$, using its definition, we have

$$
\boldsymbol{p}^{t}\left(\lambda \boldsymbol{F}_{+}-\boldsymbol{A}\right) \boldsymbol{p}=0
$$

Using (13), we have

$$
\lambda=\left(\boldsymbol{p}^{t} \boldsymbol{A} p\right) /\left(\boldsymbol{p}^{t} \boldsymbol{F}_{+} \boldsymbol{p}\right) .
$$

Using (14) and $1 \ll C_{i}$, we have $|\lambda| \ll 1$.

Here, we define vector $\boldsymbol{w}$ as $\boldsymbol{w}=(\boldsymbol{P} \boldsymbol{T})^{-1} \boldsymbol{v}_{+}$. Using (9), we have

$$
\boldsymbol{T}^{-1} \boldsymbol{P}^{t}\left(\boldsymbol{F}_{+} \boldsymbol{P} \boldsymbol{T} \Delta \boldsymbol{w}-\boldsymbol{A P T} \boldsymbol{w}\right)=0 .
$$

Using (11) and (15), we have

$$
\Delta \boldsymbol{w}-\boldsymbol{J} \boldsymbol{w}=0 .
$$

Here, we define $\boldsymbol{w}^{\prime}$ as $\boldsymbol{w}^{\prime}=\boldsymbol{w}+\Delta \boldsymbol{w}$. Then, using (16), we have

$$
\boldsymbol{w}^{\prime}=\boldsymbol{G} \boldsymbol{w}, \text { where } \boldsymbol{G}=\boldsymbol{I}+\boldsymbol{J} .
$$

The eigen value of $\boldsymbol{G}$ is $1+\alpha_{i}$. If $\left|1+\alpha_{i}\right|>1, \boldsymbol{G}^{n}$ becomes infinite so that $\boldsymbol{w}$ (namely $V_{i}$ and $\varepsilon$ ) becomes infinite. This is contradictory to the real system. In this circuit, power supply is not AC voltage but a constant one, so that the LC circuit in this system is not resonant to the power supply. Therefore, $V_{i}$ and $\varepsilon$ can not be infinite. By the ON and OFF operation of the switching transistor, $\left|V_{i}\right|$ and $|\varepsilon|$ in this circuit is smaller than a finite value, which is not dependent on the initial state and the time length of $\Delta t$. Therefore, we can say physically that $\left|1+\alpha_{i}\right| \leqq 1$.

When $\left|1+\alpha_{i}\right|=1,1+\alpha_{i}$ is located on a circle as shown in Fig. 4 (a). In this case, we make the operation such that $C_{i} \rightarrow K \cdot C_{i}(K>1)$. From (14), this operation makes $\lambda \rightarrow 1 / K \cdot \lambda$. Therefore, using $\lambda=\alpha_{i}$, we have $\alpha_{i} \rightarrow 1 / K \cdot \alpha_{i}$, which means that $\alpha_{i}$ can be sufficiently small. As already discussed, there are no eigen values outside the circle with the radius of 1 . If the eigen value is located on the circle [P in Fig. 4(a)], it can enter the circle $\left[P^{\prime}\right.$ in Fig. $\left.4(\mathrm{~b})\right]$ by this operation so that all eigen values can exist inside the circle. This means that we can always set $\left|1+\alpha_{i}\right|<1$ by this operation. This means $\boldsymbol{G}^{n} \rightarrow 0$ so that $\left|V_{i}\right|$ and $|\varepsilon|$ become 0 . In general, $\alpha_{i}$ is complex 

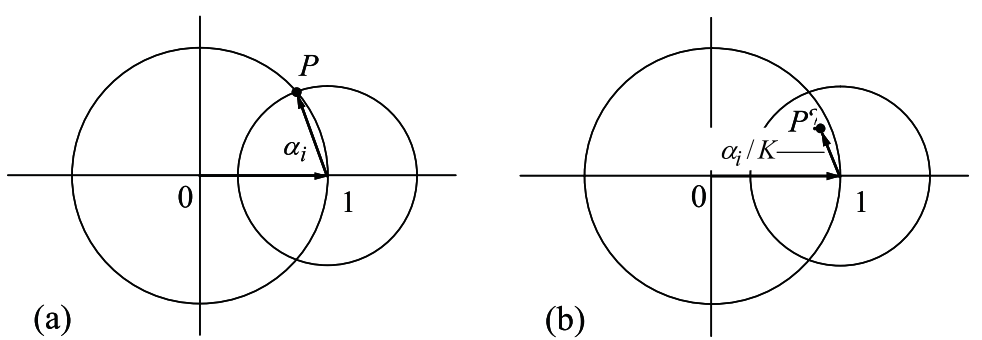

Fig. 4. Change of the eigen value with absolute value of 1. (a) Initial point. (b) Point after the operation with capacitance values larger than the initial one.

so that $\left|V_{i}\right|$ and $|\varepsilon|$ approach zero not monotonically but through oscillation as the process in Fig. 1 (c) is repeated, which is shown in Fig. 1(d) and (e).

When we use PWM, where $t_{i+1}-t_{i}$ is different in Fig. 1 (c), we can also show that the stepwise voltage is stable by simulation and the analytical method similarly. This PWM can make the waveform much closer to the ideal sinusoidal one.

\section{Conclusion}

The stability of an adiabatic circuit with inductive load was analyzed. We showed that this circuit is stable by simulation and proved its stability by an analytical method. 